\title{
COMPACT SPACES AND SPACES OF MAXIMAL COMPLETE SUBGRAPHS
}

BY

\author{
MURRAY BELL AND JOHN GINSBURG ${ }^{1}$
}

\begin{abstract}
We consider the space $M(G)$ of all maximal complete subgraphs of a graph $G$ and, in particular, the space $M(P)$ of all maximal chains of an ordered set $P$. The main question considered is the following: Which compact spaces can be represented as $M(G)$ for some graph $G$ or as $M(P)$ for some ordered set $P$ ? The former are characterized as spaces which have a binary subbase for the closed sets which consists of clopen sets. We give an example to show that this does not include all zero-dimensional supercompact spaces. The following negative result is obtained concerning ordered sets: Let $D$ be an uncountable discrete space and let $\alpha D$ denote the one-point compactification of $D$. Then there is no ordered set $P$ such that $M(P) \simeq \alpha D$.
\end{abstract}

1. Preliminaries. In this paper the term graph always refers to an undirected graph, that is, a pair $(G, E)$ where $G$ is a set and where $E$ is a symmetric subset of $G \times G$. We usually just speak of the graph $G$ when the set of edges $E$ is understood. As usual, a subset $S$ of $G$ is called a complete subgraph of $G$ if $(x, y) \in E$ for all $x, y$ in $S$, and $S$ is said to be independent if $(x, y) \notin E$ for all $x, y$ in $S$. We let $M(G)=\{C \subseteq G: C$ is a maximal complete subgraph of $G\}$. We have $M(G) \subseteq 2^{G}$ where $2^{G}$ denotes the power set of $G$. Thus $M(G)$ is also thought of as a topological space-as a subspace of $2^{G}$ with the usual product topology. Thus $M(G)$ has as a subbase for the open sets all sets of the form $A(x)=\{C \in M(G): x \notin C\}$ and $B(x)=\{C \in M(G): x \in C\}$, where $x$ is in $G$. These sets are clopen in $M(G)$ and also form a subbase for the closed subsets of $M(G)$. We note that if $C \in A(x)$ then by maximality there is an element $y$ in $C$ such that $(x, y) \notin E$. We then have $C \in B(y) \subseteq A(x)$. From this we see that the sets of the form $B(x)$ alone form a subbase for the open sets in $M(G)$.

We will be interested in two graphs naturally associated with a partially ordered set $(P, \leqslant)$. (We will use the phrase "ordered set" as synonymous with "partially ordered set".) The first of these is the comparability graph of $P$ : this is the graph $(G, E)$ where $G=P$ and where $E=\{(x, y) \in P \times P: x$ is comparable with $y\}$. (Recall that in an ordered set $P, x$ is said to be comparable with $y$ if either $x \leqslant y$ or $y \leqslant x$.) In this case we employ the usual terms chain and antichain rather than

Received by the editors March 29, 1983 and, in revised form, June 20, 1983.

1980 Mathematics Subject Classification. Primary 54D30, 05C99, 06A10.

Key words and phrases. Compact space, graph, maximal complete subgraph, binary subbase, ordered set, maximal chain, one-point compactification.

'The authors gratefully acknowledge the support of NSERC operating grants in conducting the research for this paper. The second author dedicates his research in this paper to Ezra. 
complete and independent, and we let $M(P)$ denote the space of all maximal chains of $P$ with the above topology. We will also consider the compatibility graph of $P$ : this is the graph $(G, E)$ where $G=P$ and where $E=\{(x, y) \in P \times P: x$ is compatible with $y\}$. The phrase " $x$ is compatible with $y$ " here means that there exists an element $z$ in $P$ such that $x \leqslant z$ and $y \leqslant z$. We will use the notation $M\left(G_{P}\right)$ to denote the space of maximal complete subgraphs of the compatibility graph of $P$.

Although our interest in this paper lies in the spaces $M(G)$, we note that another natural object of study is the space $C(G)$ of all complete subgraphs of $G$. The properties of $C(G)$ are discussed and applied in [1]. In this paper our main interest is in the following question: which compact spaces can be represented in the form $M(G)$ for some graph $G$ or as $M(P)$ for some ordered set $P$ ?

In general, $M(G)$ and $M(P)$ are noncompact spaces. In this section we will give simple characterizations of those graphs $G$ and ordered sets $P$ for which $M(G)$ and $M(P)$ are compact. In $\S 2$ we will consider compact spaces which can be represented in the form $M(G)$ and in $\S 3$ we will consider the situation for ordered sets $P$.

Our set-theoretic notation and terminology are standard. In particular, $\omega_{1}$ denotes the first uncountable cardinal and $|S|$ denotes the cardinality of the set $S$. A basic reference for the set-theoretic and topological concepts used in this paper is [5].

If $(G, E)$ is a graph, a subset $T$ of $G$ will be called a transversal for $G$ if $T$ intersects every maximal complete subgraph of $G$. (We also use the same term for a subset of an ordered set $P$ which meets every maximal chain of $P$.) If $x$ is an element of $G$ we let $I(x)=\{y \in G:(x, y) \notin E\}$. Thus, for an ordered set $P$ (that is, the comparability graph of $P), I(x)$ is the set of all elements of $P$ which are incomparable with $x$.

1.1 THEOREM. Let $G$ be a graph. The following statements are equivalent:

(i) $M(G)$ is compact.

(ii) Every transversal of $G$ contains a finite transversal.

(iii) For every $x$ in $G$ there is a finite set $F \subseteq I(x)$ such that $\{x\} \cup F$ is a transversal for $G$.

Proof. (i) $\rightarrow$ (ii). Let $T$ be a transversal for $G$. Then $\{B(x): x \in T\}$ is an open cover of $M(G)$, which has a finite subcover by (i). If $T^{\prime}$ is a finite subset of $T$ such that $\left\{B(x): x \in T^{\prime}\right\}$ covers $M(G)$, then $T^{\prime}$ is a finite transversal contained in $T$.

(ii) $\rightarrow$ (iii). We apply (ii) to the transversal $\{x\} \cup I(x)$.

(iii) $\rightarrow$ (i). Assuming (iii) we show that $M(G)$ is closed in $2^{G}$. Let $S_{0} \in 2^{G}-M(G)$. If $S_{0}$ is not complete then there are elements $x, y$ in $S_{0}$ with $(x, y) \notin E$. Therefore the set $U=\left\{S \in 2^{G}: x \in S\right.$ and $\left.y \in S\right\}$ is an open set in $2^{G}$ which contains $S_{0}$ and is disjoint from $M(G)$. If $S_{0}$ is complete, then since $S_{0} \notin M(G)$, there exists an element $x_{0}$ in $G-S_{0}$ such that $\left(x_{0}, y\right)$ is an edge of $G$ for all $y$ in $S_{0}$. Let $F$ be a finite subset of $I\left(x_{0}\right)$ such that $\left\{x_{0}\right\} \cup F$ is a transversal. Then the set $V=\left\{S \in 2^{G}\right.$ : $x_{0} \notin S$ and $p \notin S$ for all $p$ in $\left.F\right\}$ is an open set in $2^{G}$ which contains $S_{0}$ and is disjoint from $M(G)$. This proves that $M(G)$ is closed in $2^{G}$.

Conditions (ii) and (iii) in 1.1. are, of course, very restrictive. One notable consequence of these conditions in an ordered set $P$ concerns the nature of suprema and infima of chains. 
1.2 Theorem. Let $P$ be an ordered set and suppose $M(P)$ is compact. Let $C$ be a nonempty chain in $P$ which has a supremum in $P$, and let $u=\sup C$. Then there exists an element $p$ in $C$ such that, if $x \in P$ and $x \geqslant p$ then $x$ is comparable to $u$. The dual statement holds for infimum.

Proof. Let $F$ be a finite set of elements which are incomparable to $u$ such that $\{u\} \cup F$ meets every maximal chain of $P$. Now for each $f$ in $F, f$ is incomparable to $u$ and so there exists an element $p_{f}$ in $C$ such that $f$ is incomparable to $p_{f}$. Let $p$ be the largest of the elements $p_{f}$ for $f$ in $F$. We claim that $p$ satisfies the conclusion of the theorem, that is, if $x$ is any element of $P$ for which $x \geqslant p$, then $x$ is comparable to $u$. For, given any such $x$, let $D$ be any maximal chain in $P$ which contains the set $\{x\} \cup\left\{p_{f}: f \in F\right\}$. Then $D$ must meet $\{u\} \cup F$, but since $p_{f}$ and $f$ are incomparable $D$ must be disjoint from $F$. Therefore, we must have $u \in D$ and so $x$ and $u$ are comparable.

If the sups and infs of chains in $P$ satisfy the conclusion of 1.2 , let us say that the sups and infs in $P$ are special. This property of sups and infs can be used to give an alternate characterization of the compactness of $M(P)$ for chain-complete ordered sets. (Recall that an ordered set $P$ is said to be chain-complete if every chain in $P$ has a sup and inf in $P$.) Note that in considering $M(P)$ we can assume without loss of generality that $P$ has a 0 and 1 , since adding on these universal elements results in an ordered set $P^{\prime}$ for which $M\left(P^{\prime}\right) \simeq M(P)$. So all ordered sets considered will be assumed to have a 0 and 1 . By an open interval of $P$ we mean, as usual, a subset of $P$ of the form $(a, b)=\{x \in P: a<x<b\}$ where $a$ and $b$ are elements of $P$ with $a<b$.

1.3 TheOREM. Let $P$ be a chain-complete ordered set. Then the following are equivalent:

(i) $M(P)$ is compact.

(ii) Every nonempty open interval in $P$ has a finite transversal and all sups and infs of chains in $P$ are special.

Proof. (i) $\rightarrow$ (ii). Assume $M(P)$ is compact. We need only show, by 1.2 , that every nonempty open interval of $P$ has a finite transversal. Given a nonempty open interval $(a, b)$ of $P$, we observe that the set $\{C \in M(P): a \in C$ and $b \in C\}=B(a)$ $\cap B(b)$ is a closed subset of $M(P)$ and is hence compact. Now the space $M((a, b))$ is a continuous image of the space $B(a) \cap B(b)$ under the function which maps $C$ to $C \cap(a, b)$ and so $M((a, b))$ is compact. Therefore $(a, b)$ has a finite transversal by 1.1 .

(ii) $\rightarrow$ (i). Assume (ii). We show that $M(P)$ is closed in $2^{P}$. Let $S_{0}$ be any element of $2^{P}-M(P)$. We show there is an open set in $2^{P}$ containing $S_{0}$ which is disjoint from $M(P)$. If $S_{0}$ is not a chain in $P$ this is easily done, as in the proof of (iii) $\rightarrow$ (i) of 1.1. Furthermore, if either 0 or 1 does not belong to $S_{0}$ then $\left\{S \in 2^{P}: 0 \notin S\right\}$ or $\left\{S \in 2^{P}: 1 \notin S\right\}$, respectively, is an open set in $2^{P}$ containing $S_{0}$ which is disjoint from $M(P)$. So we assume that $S_{0}$ is a chain in $P$ containing both 0 and 1 . Then since $S_{0}$ is not in $M(P)$ there is an element $u \in P-S_{0}$ such that $S_{0} \cup\{u\}$ is a chain. Let $C=\left\{x \in S_{0}: x<u\right\}$ and let $D=\left\{x \in S_{0}: u<x\right\}$. Note that $C$ and $D$ are 
nonempty and $C \cup D=S_{0}$. Let $c=\sup C$ and let $d=\inf D$. Then $c \leqslant u \leqslant d$. Now, if both $c$ and $d$ are in $S_{0}$ then $(c, d)$ is a nonempty open interval of $P$ which is disjoint from $S_{0}$. By (ii), $(c, d)$ has a finite transversal $T$. But then the set $U=\left\{S \in 2^{P}: c \in S\right.$ and $d \in S$ and $x \notin S$ for all $x$ in $\left.T\right\}$ is an open set in $2^{P}$ containing $S_{0}$ which is disjoint from $M(P)$ as desired. If either $c$ or $d$ is not in $S_{0}$ we argue as follows: say $c$ is not in $S_{0}$. (If $d$ is not in $S_{0}$ we argue dually.) Since sups in $P$ are special there is an element $p$ in $C$ such that if $x \in P$ and $x \geqslant p$ then $x$ is comparable with $c$. This obviously implies that any maximal chain containing $p$ must also contain $c$. Therefore the set $V=\left\{S \in 2^{P}: p \in S\right.$ and $\left.c \notin S\right\}$ is an open set in $2^{P}$ containing $S_{0}$ which is disjoint from $M(P)$. This shows that $M(P)$ is closed in $2^{P}$ and hence compact.

In [4], ordered sets in which every element lies in a finite transversal as in 1.1 are examined, particularly as to the size of antichains in such ordered sets.

To conclude this section we observe that any space of maximal complete subgraphs can actually be obtained from a compatibility graph.

1.4 THEOREM. For any graph $G$ there is an ordered set $P$ such that $M(G) \simeq M\left(G_{P}\right)$ where $G_{P}$ is the compatibility graph of $P$.

Proof. Let $P=\{S \subseteq G: S$ is finite and complete $\}$ be ordered by inclusion. Thus $G_{P}$ is the graph having $P$ as set of vertices, and if $S_{1}$ and $S_{2}$ are in $P$ then $S_{1}$ and $S_{2}$ are joined by an edge of $G_{P}$ if and only if $S_{1}$ and $S_{2}$ are compatible in $(P, \subseteq)$; this is clearly equivalent to the statement that $S_{1} \cup S_{2}$ is complete. It is easily checked that if $C \in M(G)$ then the collection $\gamma=\{S \in P: S \subseteq C\}$ is a maximal complete subgraph of $G_{P}$. Conversely, if $\gamma$ is a maximal complete subgraph of $G_{P}$ then $C=\cup_{\gamma}$ is a maximal complete subgraph of $G$. This sets up a bijection $f$ from $M(G)$ onto $M\left(G_{P}\right)$. Under this mapping we have that $f(B(x))=B(\{x\})$ for any $x$ in $G$ and $f^{-1}(B(S))=\bigcap\{B(x): x \in S\}$ for any $S$ in $P$. Therefore, $f$ is a homeomorphism.

2. Compact spaces as spaces of maximal complete subgraphs. We now characterize which compact spaces can be represented in the form $M(G)$. First we recall that a collection of sets $s$ is said to be binary if the following condition holds: if $s^{\prime} \subseteq s$ and if very pair of sets in $s^{\prime}$ has nonempty intersection, then $\cap s^{\prime} \neq \varnothing$. We also recall that a topological space $X$ is said to be supercompact if there is a binary subbase for the closed subsets of $X$. (This property is stronger than compactness and has been extensively studied; a basic reference is [6].)

2.1 THEOREM. Let $X$ be a compact space. Then the following are equivalent:

(i) There is a graph $G$ such that $X \simeq M(G)$.

(ii) $X$ has a binary subbase for the closed sets consisting of clopen sets.

Proof. (i) $\rightarrow$ (ii). Suppose $X$ is compact and that $X \simeq M(G)$ for some graph $G$. Since the collection of clopen sets $s=\{B(x): x \in G\}$ is clearly binary, it is sufficient to show that $s$ is a subbase for the closed sets in $M(G)$ if $M(G)$ is compact. But this follows easily: let $F$ be any closed subset of $M(G)$. If $C \in M(G)-F$ then, since $s$ does form a subbase for the open sets in $M(G)$, we can cover $F$ by a collection of sets of the form $B(x)$, none of which contain $C$. Since $F$ is compact, a 
finite subcollection of these cover $F$. This shows that $F$ is an intersection of finite unions of members of $s$, as desired.

(ii) $\rightarrow$ (i). Suppose $X$ has a binary subbase $s$ for the closed sets which consists of clopen sets. Let $G$ be the intersection graph of $s$. Thus the set of vertices of $G$ is $s$ and, for $S_{1}$ and $S_{2}$ in $s,\left(S_{1}, S_{2}\right)$ forms an edge in $G$ if and only if $S_{1} \cap S_{2} \neq \varnothing$. We claim that $X \simeq M(G)$. This is seen as follows. If $\gamma \in M(G)$ then every pair of sets in $\gamma$ intersect, and so, since $s$ is binary, $\cap \gamma \neq \varnothing$. Let $x$ be an element of $\cap \gamma$, and let $\gamma_{x}=\{S \in s: x \in S\}$. We have $\gamma \subseteq \gamma_{x}$ and so by maximality $\gamma=\gamma_{x}$. Now, we may assume without loss of generality that the subbase $s$ is closed under finite intersections. A simple compactness argument now shows that, for each $x$ in $X$, the collection $\gamma_{x}$ defined above is a maximal complete subgraph of $G$. Since $s$ is a subbase for the closed sets we have that $\gamma_{x} \neq \gamma_{y}$ if $x \neq y$ and hence the function $f$ from $X$ to $M(G)$ defined by $f(x)=\gamma_{x}$ is a bijection. For any subbasic open set $B(S)$ in $M(G)$, where $S \in s$, we have $f^{-1}(B(S))=\left\{x \in X: S \in \gamma_{x}\right\}=S$, which is open in $X$ since each $S$ in $s$ is clopen. It follows that $f$ is a homeomorphism.

It is immediate that any compact space $X$ which can be represented in the form $M(G)$ must be zero-dimensional and supercompact. It is indeed natural to conjecture that the spaces which satisfy (ii) in 2.1 are precisely the zero-dimensional supercompact spaces. This is not true, however, as we will presently show. We note that the most familiar zero-dimensional supercompact spaces do indeed satisfy the condition (ii) above - the spaces $\{0,1\}^{\kappa}$ for any cardinal $\kappa$; the linearly ordered space $\alpha+1$ for any ordinal $\alpha$; one-point compactifications of discrete spaces; the "double-arrow" space, and so on.

2.2 EXAMPLE. A zero-dimensional supercompact space which has no binary subbase for the closed sets which consists of clopen sets.

Let $X=\left\{S: S \subseteq \omega_{1}\right.$ and $\left.|S| \leqslant 2\right\} . X$ is a subspace of $2^{\omega_{1}}$. We will show that $X$ has the desired properties. $X$ is clearly closed in $2^{\omega_{1}}$ and hence compact. Note that if $\alpha<\beta<\omega_{1}$ then $\{\alpha, \beta\}$ is an isolated point of $X$. If $\alpha<\omega_{1}$ and if $F$ is a finite subset of $\omega_{1}$, we let $B_{\alpha}(F)=\{\{\alpha\}\} \cup\left\{\{\alpha, \beta\}: \beta \in \omega_{1}-F\right\}$. The family $\left\{B_{\alpha}(F): F\right.$ is a finite subset of $\left.\omega_{1}\right\}$ is a neighborhood base in $X$ for the point $\{\alpha\}$. Also for each finite subset $F$ of $\omega_{1}$ we let $B(F)=\{S \in X: S \cap F=\varnothing\}$. The collection $\{B(F): F$ is a finite subset of $\left.\omega_{1}\right\}$ is a neighborhood base in $X$ for the point $\varnothing$. Note that the sets $B(F)$ and $B_{\alpha}(F)$ are clopen in $X . X$ is evidently zero-dimensional, and we now establish the stated properties in two claims.

Claim 1. $X$ is supercompact. To prove this, we introduce the following notation. For each $\alpha^{*}<\omega_{1}$ and each finite subset $F$ of $\omega_{1}$, we let $L_{\alpha}(F)=\{\{\alpha\}\} \cup\{\{\alpha, \beta\}$ : $\beta \in \omega_{1}-F$ and $\left.\beta<\alpha\right\}$ and we let $R_{\alpha}(F)=\{\{\alpha\}\} \cup\left\{\{\alpha, \beta\}: \beta \in \omega_{1}-F\right.$ and $\beta>\alpha\}$. Note that $B_{\alpha}(F)=L_{\alpha}(F) \cup R_{\alpha}(F)$ and $L_{\alpha}(F) \cap R_{\alpha}(F)=\{\{\alpha\}\}$. Now set $s_{1}=\left\{\{\{\alpha, \beta\}\}: \alpha<\beta^{*}<\omega_{1}\right\}, s_{2}=\left\{L_{\alpha}(F): \alpha^{*}<\omega_{1}\right.$ and $F$ is a finite subset of $\left.\omega_{1}\right\}$, $s_{3}=\left\{R_{\alpha}(F): \alpha^{\circ}<\omega_{1}\right.$ and $F$ is a finite subset of $\left.\omega_{1}\right\}$ and $s_{4}=\{B(F): F$ is a finite subset of $\left.\omega_{1}\right\}$. Finally let $s=s_{1} \cup s_{2} \cup s_{3} \cup s_{4}$. We show that $s$ is a binary subbase for the closed subsets of $X$.

Now $s$ consists of closed subsets of $X$ and, making use of the neighborhood bases mentioned above, it is easily checked that if $C$ is a closed subset of $X$ and 
$S \in X-C$, then there exists a finite $s^{\prime} \subseteq s$ such that $C \subseteq \cup s^{\prime}$ and $S \notin \cup s^{\prime}$. Therefore $s$ is a subbase for the closed sets in $X$. To show that $s$ is binary it suffices to show (because $X$ is compact) that if $s^{\prime}$ is a finite subcollection of $s$ such that every pair of sets in $s^{\prime}$ intersect, then $\cap s^{\prime} \neq \varnothing$. Let $s^{\prime}$ be such a finite subcollection of $s$. If $s^{\prime} \cap s_{1} \neq \varnothing$, then clearly $\cap s^{\prime} \neq \varnothing$. So we may assume that $s^{\prime}$ contains no sets from $s_{1}$. Now if $\alpha \neq \beta$ then $L_{\alpha}(F) \cap L_{\beta}(G)=\varnothing$ and $R_{\alpha}(F) \cap R_{\beta}(G)=\varnothing$ for any $F$ and $G$. Therefore, $s^{\prime}$ must be of the form

$$
s^{\prime}=\left\{L_{\alpha}\left(F_{i}\right): i<m\right\} \cup\left\{R_{\beta}\left(G_{i}\right): i<n\right\} \cup\left\{B\left(H_{i}\right): i<r\right\},
$$

where $m, n$ and $r$ are positive integers, and where any (but not all) of the three displayed subcollections may not be present. If $\alpha=\beta$ then clearly $\{\alpha\} \in \cap s^{\prime}$. If $\alpha \neq \beta$ then $\beta<\alpha$ and clearly $\{\alpha, \beta\} \in \cap s^{\prime}$. (Here we are assuming that the first two subcollections displayed in $s^{\prime}$ are, in fact. present.) If the first subcollection is not present (but the second is) then $\{\beta\} \in \cap s^{\prime}$. If the second is not present (but the first is) then $\{\alpha\} \in \cap s^{\prime}$. If neither the first or second displayed subcollection is present then clearly $\cap s^{\prime} \neq \varnothing$. This shows that $s$ is binary, as desired.

Claim 2. $X$ does not have a binary subbase for the closed sets which consists of clopen sets: To show this, let $s$ be any subbase for the closed sets in $X$ which consists of clopen sets. We will show that $s$ is not binary. Let $s^{\prime}$ be the collection of all finite intersections of members of $s$. Then $s^{\prime}$ is also a subbase for the closed sets, which is furthermore closed under finite intersections. A standard compactness argument shows that every clopen subset of $X$ is a union of finitely many members of $s^{\prime}$. Thus for each $\alpha<\omega_{1}$ there exists a finite $f_{\alpha} \subseteq s^{\prime}$ such that $B_{\alpha}(\varnothing)=\cup f_{\alpha}$. For each $\alpha$ choose a set $S_{\alpha} \in f_{\alpha}$ such that $\{\alpha\} \in S_{\alpha}$. Since $S_{\alpha}$ is open, there exists a finite set $F_{\alpha}$ such that $\{\alpha\} \in B_{\alpha}\left(F_{\alpha}\right) \subseteq S_{\alpha}$. Consider the family of finite sets $\left\{F_{\alpha}: \alpha \in \omega_{1}\right\}$. By the Free Set Lemma (see [5, p. 96], for example), there surely exist three distinct ordinals $\alpha, \beta, \gamma$ such that $\alpha \notin F_{\beta} \cup F_{\gamma}, \beta \notin F_{\alpha} \cup F_{\gamma}$ and $\gamma \notin F_{\alpha} \cup F_{\beta}$. Now, any two sets from the family $\left\{S_{\alpha}, S_{\beta}, S_{\gamma}\right\}$ intersect (e.g. $\left.S_{\alpha} \cap S_{\beta} \supseteq B_{\alpha}\left(F_{\alpha}\right) \cap B_{\beta}\left(F_{\beta}\right) \supset\{\{\alpha, \beta\}\}\right)$. But $S_{\alpha} \cap S_{\beta} \cap S_{\gamma} \subseteq B_{\alpha}(\varnothing) \cap B_{\beta}(\varnothing) \cap B_{\gamma}(\varnothing)=\varnothing$. Therefore $s^{\prime}$ is not binary and so $s$ is not binary.

3. Compact spaces as spaces of maximal chains. In this section we consider the compact spaces which can be represented in the form $M(P)$ for some ordered set $P$. A characterization of these spaces analogous to 2.1 can be given using the well-known characterization of comparability graphs from [3]; in this case one needs a binary subbase for the closed sets which consists of clopen sets and whose intersection graph is a comparability graph. However, such a description is not particularly interesting topologically, and we will not pursue it here. We do not know of any useful topological property which distinguishes such spaces from the spaces in 2.1. However we can make several observations: For any cardinal $\kappa$, the space $\{0,1\}^{\kappa}$ can be represented in the form $M(P)$. $P$ can be taken to be the ordinal sum of $\kappa$ copies of the three element ordered set $\{a, b, c\}$ in which $a<b, a<c$, and $b$ and $c$ are incomparable. For any ordinal $\lambda$, the compact linearly ordered space $\lambda+1$ can be represented in the form $M(P)$. Here we can take $P$ to be the union of two copies of the chain $\lambda$, say $P=\lambda \times\{0,1\}$, ordered as follows: each copy of $\lambda-\lambda \times\{0\}$ and 
$\lambda \times\{1\}$, retains its usual ordering, and we set $(\xi, 0)<(\eta, 1)$ iff $\xi<\eta$. A similar ordered set can be used to represent the compact linearly ordered space $C \times\{0,1\}$, ordered lexicographically, where $C$ is any complete chain. (In particular, this includes the double-arrow space, when $C$ is a closed interval in the reals.)

We also note that the class of compact spaces which can be represented in the form $M(P)$ is closed under products. This follows from the observation that, if $P=\bigcup\left\{P_{\alpha}: \alpha<\kappa\right\}$ is the ordinal sum of the ordered sets $P_{\alpha}$ (that is, all the elements of $P_{\alpha}$ precede all the elements of $P_{\beta}$ if $\alpha^{*}<\beta$, while each $P_{\alpha}$ retains its own ordering), then $M(P)$ is homeomorphic to the topological product of the spaces $M\left(P_{\alpha}\right)$.

In fact, it is only with some difficulty that we are able to exhibit a compact space which can be represented in the form $M(G)$ for some graph $G$ but not in the form $M(P)$ for any ordered set $P$. As we will now show, if $D$ is an uncountable discrete space and if $\alpha D$ denotes the one-point compactification of $D$, then $\alpha D$ cannot be represented in the form $M(P)$ for any ordered set $P$. By $2.1, \alpha D$ can be represented in the form $M(G)$ for some graph $G$. This also can be seen directly by considering the graph $G$ having as set of vertices the set $D \times\{0,1\}$, in which $D \times\{0\}$ is a complete subgraph, $D \times\{1\}$ is independent, and there is an edge joining $(x, 0)$ to $(y, 1)$ if and only if $x \neq y$. Thus by 1.4 we will have that $\alpha D$ can be represented as the space of maximal complete subgraphs of the compatibility graph of an ordered set, but not from the comparability graph of an ordered set.

In the following discussion, if $S$ and $T$ are subsets of an ordered set $P$ we write $S<T$ to indicate that $x<y$ for all $x$ in $S$ and all $y$ in $T$.

3.1 Theorem. Let $D$ be an uncountable discrete space, and let $\alpha D$ denote the one-point compactification of $D$. There is no ordered set $P$ such that $M(P) \simeq \alpha D$.

We will assume that such an ordered set $P$ does exist and derive a contradiction. We will make use of two lemmas.

3.2 Lemma. Suppose $D$ is an uncountable discrete space and $P$ is an ordered set such that $M(P) \simeq \alpha D$. Then $P$ contains no uncountable antichains.

Proof. Let $L$ be the "point at infinity" in $M(P)$. Thus every neighborhood of $L$ in $M(P)$ contains all but a finite number of the maximal chains in $M(P)$, and every other maximal chain of $P$ is an isolated point of $M(P)$. Suppose $P$ does contain an uncountable antichain $A=\left\{a_{\alpha}: \alpha<\omega_{1}\right\}$. For each $\alpha$, choose a maximal chain $C_{\alpha}$ in $P$ such that $a_{\alpha} \in C_{\alpha}$. Now for each $x$ in $L$ we have $L \in B(x)$ and so $C_{\alpha} \in B(x)$ except for a finite set of $\alpha$ 's. That is, $x$ is in $C_{\alpha}$ for all but a finite number of $\alpha$, and so $x$ is comparable to $a_{\alpha}$ for all but finitely many $\alpha$. Since $A$ is an antichain, this means that either the set $x^{+}=\left\{\alpha \in \omega_{1}: a_{\alpha}<x\right\}$ is a cofinite subset of $\omega_{1}$ or the set $x^{-}=\left\{\alpha \in \omega_{1}: x<a_{\alpha}\right\}$ is a cofinite subset of $\omega_{1}$. We let $L^{+}=\left\{x \in L: x^{+}\right.$is a cofinite subset of $\left.\omega_{1}\right\}$ and $L^{-}=\left\{x \in L: x^{-}\right.$is a cofinite subset of $\left.\omega_{1}\right\}$. We have $L=L^{+} \cup L^{-}$and $L^{-}<L^{+}$. We now consider four possible cases according to whether or not $L^{-}$has a countable cofinal subset and whether or not $L^{+}$has a countable coinitial subset. 
Case 1. $L^{-}$has a countable cofinal subset $\left\{x_{n}: n \in \omega\right\}$ and $L^{+}$has a countable coinitial subset $\left\{y_{n}: n \in \omega\right\}$ : In this case we note that the set

$$
W=\left[\cap\left\{x_{n}^{-}: n \in \omega\right\}\right] \cap\left[\cap\left\{v_{n}^{+}: n \in \omega\right\}\right]
$$

is a cocountable subset of $\omega_{1}$. Let $\alpha$ be any element of $W$. Then $x_{n}<a_{\alpha}<y_{n}$ for all $n$ and hence $L^{-}<a_{\alpha}<L^{+}$. But this contradicts the maximality of $L$, since $L=L^{-}$ $\cup L^{+}$.

Case 2. $L^{-}$has a countable cofinal subset $\left\{x_{n}: n \in \omega\right\}$ while $L^{+}$has no countable coinitial subset: In this case, let $W=\bigcap\left\{x_{n}^{-}: n \in \omega\right\}$. Then $W$ is a cocountable subset of $\omega_{1}$ and $L^{-}<a_{\alpha}$ for all $\alpha$ in $W$. Let $S$ be any countable, infinite subset of $W$. By the maximality of $L$ for no $\alpha$ in $S$ is it true that $a_{\alpha}<L^{+}$. Thus for each $\alpha$ in $S$ there is an element $p_{\alpha}$ in $L^{+}$such that $a_{\alpha}$ is not $<p_{\alpha}$. By our assumption in Case 2 , the set $\left\{p_{\alpha}: \alpha \in S\right\}$ cannot be coinitial in $L^{+}$, and so there is an element $p$ in $L^{+}$ such that $p<p_{\alpha}$ for all $\alpha$ in $S$. Since $p$ is in $L^{+}$, the set $p^{+}$is cofinite and so $p^{+}$must meet $S$. But for any $\alpha \in p^{+} \cap S$ we have $a_{\alpha}<p<p_{\alpha}$ contrary to the choice of $p_{\alpha}$.

Case 3. $L^{+}$has a countable coinitial subset and $L^{-}$has no countable cofinal subset: Here we use the dual argument to Case 2.

Case 4. $L^{+}$has no countable coinitial subset and $L^{-}$has no countable cofinal subset: In this case we let $E_{1}=\left\{\alpha \in \omega_{1}: a_{\alpha}<L^{+}\right\}, E_{2}=\left\{\alpha \in \omega_{1}: a_{\alpha}>L^{-}\right\}$and $E_{3}=\omega_{1}-\left(E_{1} \cup E_{2}\right)$. Now one of these three sets is infinite. If either $E_{1}$ or $E_{2}$ is infinite we argue as in Cases 2 and 3 above. So suppose $E_{3}$ is infinite. Then let $S$ be a countable, infinite subset of $E_{3}$. For each $\alpha$ in $S$ there are elements $x_{\alpha}$ in $L^{-}$and $y_{\alpha}$ in $L^{+}$such that $x_{\alpha}$ is not less than $a_{\alpha}$, and $a_{\alpha}$ is not less than $y_{\alpha}$. Now $\left\{x_{\alpha}: \alpha \in S\right\}$ is not cofinal in $L^{-}$and $\left\{y_{\alpha}: \alpha \in S\right\}$ is not coinitial in $L^{+}$, so there are elements $p \in L^{-}$and $q \in L^{+}$such that $x_{\alpha}<p<q<y_{\alpha}$ for all $\alpha$ in $S$. But then the set $p^{-} \cap q^{+}$is a cofinite subset of $\omega_{1}$ which cannot meet $S$, a contradiction. This completes the proof of Lemma 3.2.

3.3 LEMma. Let $D$ be an uncountable discrete space and let $P$ be an ordered set such that $M(P) \simeq \alpha D$. Then every uncountable subset of $P$ contains an uncountable chain.

Proof. Let $A$ be any uncountable subset of $P$. As above, let $L$ be the point at infinity in $M(P)$. If $A \cap L$ is uncountable, we are done. So we may assume without loss of generality that $A \cap L=\varnothing$ (otherwise replace $A$ by $A-L$ ). Now, by 3.2, $A$ contains no uncountable antichains, and so there must exist an element $a$ in $A$ such that the set $C(a)=\{x \in A: x$ is comparable to $a\}$ is uncountable. (If this were not the case then, by a familiar argument as in [2], we could inductively choose elements $a_{\alpha}$ in $A$ for $\alpha<\omega_{1}$ such that $a_{\alpha} \notin \cup\left\{C\left(a_{\beta}\right): \beta<\alpha\right\}$, resulting in an uncountable antichain $\left\{a_{\alpha}: \alpha<\omega_{1}\right\}$.) To be specific, suppose that the set $C^{\prime}(a)=\{x \in A$ : $a \leqslant x\}$ is uncountable. (If the set $C^{\prime \prime}(a)$ of all elements of $A$ which are $\leqslant a$ is uncountable, the argument is similar.) Now if there are only a finite number of maximal chains in the ordered set $C^{\prime}(a)$, then clearly one of them must be uncountable and so we are done. So we may as well assume that there are infinitely many maximal chains in $C^{\prime}(a)$. Extending these to maximal chains in $P$, we obtain an infinite number of maximal chains in $P$ which contain $a$. Thus the clopen set 
$B(a)=\{C \in M(P): a \in C\}$ is infinite. Therefore we must have $L \in B(a)$, for otherwise $M(P)-B(a)$ would be a neighborhood of $L$ which does not contain all but a finite number of the points of $M(P)$. We thus have that $a \in L$, contrary to our assumption that $A \cap L=\varnothing$. So, in fact, there must only be a finite number of maximal chains in $C^{\prime}(a)$, and we are done.

Proof of Theorem 3.1. Let $P$ be an ordered set for which $M(P) \simeq \alpha D$, where $D$ is an uncountable discrete space. As above, let $L$ be the point at infinity in $M(P)$. Every other point of $M(P)$ is an isolated point in $M(P)$. For any finite subset $F$ of $P$, we let $B(F)=\bigcap\{B(x): x \in F\}$. Thus $B(F)=\{C \in M(P): F \subseteq C\}$. The sets $B(F)$, for $F$ finite, form a basis for the open sets in $M(P)$. We note that $B(F)$ is nonempty if and only if $F$ is a chain, and that $B\left(F_{1}\right) \cap B\left(F_{2}\right) \neq \varnothing$ if and only if $F_{1} \cup F_{2}$ is a chain. Now, let $\left\{C_{\alpha}: \alpha<\omega_{1}\right\}$ be an uncountable set of isolated points in $M(P)$. Thus for each $\alpha$ there is a finite set $F_{\alpha}$ such that $B\left(F_{\alpha}\right)=\left\{C_{\alpha}\right\}$. By passing to a subsequence if necessary, we may assume that there is a positive integer $n$ such that $\left|F_{\alpha}\right|=n$ for all $\alpha$. Thus $F_{\alpha}$ is an $n$-element chain contained in $C_{\alpha}$. Since $\alpha \neq \beta \rightarrow$ $B\left(F_{\alpha}\right) \cap B\left(F_{\beta}\right)=\varnothing$, we have that $F_{\alpha} \cup F_{\beta}$ is not a chain if $\alpha \neq \beta$, and so some elements of $F_{\alpha}$ is incomparable with some element of $F_{\beta}$.

To prove 3.1 we now prove, by induction on $n$, the following statement: There is no ordered set $P$ such that $M(P) \simeq \alpha D$ and in which there exists a collection of finite chains $\left\{F_{\alpha}: \alpha<\omega_{1}\right\}$ with $\left|F_{\alpha}\right|=n$ for all $\alpha<\omega_{1}$, and such that $F_{\alpha} \cup F_{\beta}$ is not a chain if $\alpha \neq \beta$. The case $n=1$ is taken care of by Lemma 3.2. Assume it is true for $n$. Suppose we then had an ordered set $P$ for which $M(P) \simeq \alpha D$ and a family $\left\{F_{\alpha}\right.$ : $\left.\alpha<\omega_{1}\right\}$ of chains in $P$ with $\left|F_{\alpha}\right|=n+1$ for each $\alpha$ and such that $F_{\alpha} \cup F_{\beta}$ is not a chain if $\alpha \neq \beta$. Let us display the elements of $F_{\alpha}$ as $F_{\alpha}=\left\{x_{\alpha, 1}, x_{\alpha, 2}, \ldots, x_{\alpha, n+1}\right\}$ where we have $x_{\alpha, 1}<x_{\alpha, 2}<\cdots<x_{\alpha, n+1}$. By Lemma 3.3 (passing to a subsequence if necessary), we may assume that the set $\left\{x_{\alpha, 1}: \alpha<\omega_{1}\right\}$ is a chain. We now show that there are at most countably many $\alpha$ for which $x_{\alpha, 1} \notin L$. For, suppose the set $R=\left\{\alpha<\omega_{1}: x_{\alpha, 1} \notin L\right\}$ is uncountable. A simple argument then shows there is an element $\alpha_{0}$ in $R$ for which the set $S=\left\{\beta \in R: x_{\alpha_{0,1}} \leqslant x_{\beta, 1}\right\}$ is infinite. For each $\beta$ in $S, F_{\beta} \cup\left\{x_{\alpha_{0}, 1}\right\}$ is a chain and so can be extended to a maximal chain $D_{\beta}$ of $P$. Therefore $x_{\alpha_{0}, 1}$ lies in an infinite number of maximal chains $D_{\beta}$, and so the clopen set $B\left(x_{\alpha_{0}, 1}\right)$ is infinite. As in the proof of Lemma 3.3 it follows that $L \in B\left(x_{\alpha_{0}, 1}\right)$ and so $x_{\alpha_{0}, 1} \in L$. This contradicts the fact that $\alpha_{0} \in R$. This proves our claim that there are at most countably many $\alpha$ for which $x_{\alpha, 1} \notin L$. By discarding this countable set, we may assume without loss of generality that $x_{\alpha, 1} \in L$ for all $\alpha$. Choose a maximal chain $C_{\alpha}$ in $P$ such that $F_{\alpha} \subseteq C_{\alpha}$, for each $\alpha<\omega_{1}$. For each $\alpha$ we have $L \in B\left(x_{\alpha, 1}\right)$ and so $C_{\beta} \in B\left(x_{\alpha, 1}\right)$ for all but finitely many $\beta<\omega_{1}$. Note that if $C_{\beta} \in B\left(x_{\alpha, 1}\right)$ then $F_{\beta} \cup\left\{x_{\alpha, 1}\right\}$ is a chain. For each $\alpha$ let $H(\alpha)=\left\{\beta<\omega_{1}: F_{\beta} \cup\left\{x_{\alpha, 1}\right\}\right.$ is not a chain $\}$. Then $H(\alpha)$ is finite for each $\alpha<\omega_{1}$. By the Free Set Lemma (see [5]), there is an uncountable subset $W$ of $\omega_{1}$ such that for all $\alpha$ and $\beta$ in $W$ we have $\beta \notin H(\alpha)$. This means that, for all $\alpha, \beta$ in $W$, the sets $F_{\alpha} \cup\left\{x_{\beta, 1}\right\}$ and $F_{\beta} \cup\left\{x_{\alpha, 1}\right\}$ are both chains. But $F_{\alpha} \cup F_{\beta}$ is not a chain, and so we have that $\left[F_{\alpha}-\left\{x_{\alpha, 1}\right\}\right] \cup\left[F_{\beta}-\left\{x_{\beta, 1}\right\}\right]$ is not a chain. We now can apply our inductive hypothesis to the family of $n$-element chains $F_{\alpha}-\left\{x_{\alpha, 1}\right\}$ for $\alpha$ in $W$, to obtain a contradiction. This completes the proof. 
As mentioned before the proof of 3.1 , the authors have been unable to discover any property enjoyed by compact spaces representable in the form $M(P)$ which is useful in recognizing such spaces. For some specific compact spaces (such as the Alexandrov double of $\{0,1\}^{\omega}$ ) we do not know whether such a representation is possible.

\section{REFERENCES}

1. M. Bell, The space of complete subgraphs of a graph, Comment. Math. Univ. Carolin. 23 (1982), 525-536.

2. B. Dushnik and E. W. Miller, Partially ordered sets, Amer. J. Math. 63 (1941), 600-610.

3. P. C. Gilmore and A. J. Hoffman, A characterization of comparability graphs and of interval graphs, Canad. J. Math. 16 (1964), 539-548.

4. J. Ginsburg, I. Rival and W. Sands, Antichains and finite sets that meet all maximal chains (to appear).

5. I. Juhasz, Cardinal functions in topology, Math. Centre Tracts 34, Math. Centre, Amsterdam, 1971.

6. J. van Mill, Supercompactness and Wallman spaces, doctoral dissertation, Free University of Amsterdam, 1977

Department of Mathematics, University of Manitoba, Winnipeg, Manitoba R3T2N2, Canada

Department of Mathematics, University of Winnipeg, WinNipeg, Manitoba R3B2E9, Canada 